\title{
Case Report \\ Delayed Infection of a Lymphocele following RARP in a Patient with Nonspecific Symptoms
}

\author{
Tomoki Taniguchi, Yoshito Takahashi, Mitsuhiro Taniguchi, \\ Toru Yamada, and Kenichiro Ishida
}

Department of Urology, Gifu Prefectural General Medical Center, 4-6-1 Noishiki, Gifu 500-8717, Japan

Correspondence should be addressed to Tomoki Taniguchi; tomokidbx@gmail.com

Received 6 February 2017; Revised 8 March 2017; Accepted 9 March 2017; Published 15 March 2017

Academic Editor: Sung Kyu Hong

\begin{abstract}
Copyright (c) 2017 Tomoki Taniguchi et al. This is an open access article distributed under the Creative Commons Attribution License, which permits unrestricted use, distribution, and reproduction in any medium, provided the original work is properly cited.

Pelvic lymphoceles are an infrequent complication after pelvic surgery and develop shortly after the surgery in most cases. We experienced a case of delayed infection of a lymphocele 6 months after robot-assisted radical prostatectomy (RARP) and pelvic lymphadenectomy. In this case, antimicrobial chemotherapy and percutaneous drainage were effective, and there was no recurrence of the disease. Most urologists do not recognize that infected lymphoceles can develop a long time after surgery; thus, infected lymphoceles should be kept in mind in patients with nonspecific infectious symptoms, regardless of the length of time after surgery.
\end{abstract}

\section{Introduction}

Lymphocele development is known as an infrequent complication after radical prostatectomy and pelvic lymphadenectomy [1]. Most lymphoceles develop shortly after the surgery and do not become symptomatic, though some can cause such problems as pain, deep vein thrombosis, pulmonary embolism, and infection. However, asymptomatic lymphoceles can be infected several months after the surgery. Most cases of delayed infection of lymphoceles complained of no specific symptoms. Therefore, its diagnosis is usually delayed. In addition, most urologists do not recognize that infected lymphoceles can develop several months or more than 1 year after radical prostatectomy and lymphadenectomy. There have been only 4 published reports of 7 patients with delayed infection of lymphoceles after the surgery: 3 cases in which the pathogenic bacteria are Staphylococcus aureus, 1 case with S. agalactiae, and 3 cases in which the pathogenic bacteria are not mentioned [2].

\section{Case Presentation}

A 79-year-old Japanese man with a prostate-specific antigen level of $6.5 \mu \mathrm{g} / \mathrm{ml}$ presented to our department. $\mathrm{He}$ was diagnosed as having localized adenocarcinoma of the prostate, Gleason 3+3, cT2c N0 M0. He underwent transperitoneal non-nerve-sparing robot-assisted laparoscopic radical prostatectomy (RARP) with pelvic lymph node dissection, and a pelvic drain was placed for 3 days. The pathologic examination revealed that the tumor was $\mathrm{pT} 2 \mathrm{c}$ with Gleason $3+5$, and none of the dissected lymph nodes were malignant. On the 7th day after surgery, he was discharged from hospital with no complications. Six months after the operation, he visited a primary care doctor complaining of fever and fatigue. For a week, his symptoms continued. He was referred to the general medicine department in our hospital. A pelvic computed tomography (CT) scan showed a pelvic cyst, and a blood test revealed an abnormally high inflammatory reaction; he was then sent to our department and admitted.

On admission, his body temperature was $39.3^{\circ} \mathrm{C}$. On physical examination, any specific findings, excluding right lower abdominal pain, were not observed. Laboratory data revealed a CRP of $22.38 \mathrm{mg} / \mathrm{dl}$ and WBC of $12600 / \mu \mathrm{l}$. A urine test showed no abnormal findings. Abdominal ultrasonography (US) and pelvic CT scan revealed an $80 \mathrm{~mm}$ cystic lesion displacing the urinary bladder (Figure 1).

The treatment course is shown in Figure 2. We began treatment with intravenous administration of $3 \mathrm{~g}$ flomoxef per day. On the second day after admission, we performed 


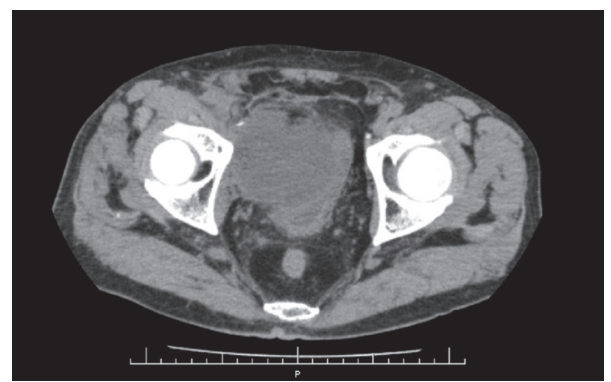

FIGURE 1: CT scan shows an $80 \mathrm{~mm}$ cystic lesion displacing the urinary bladder 6 months after the surgery.

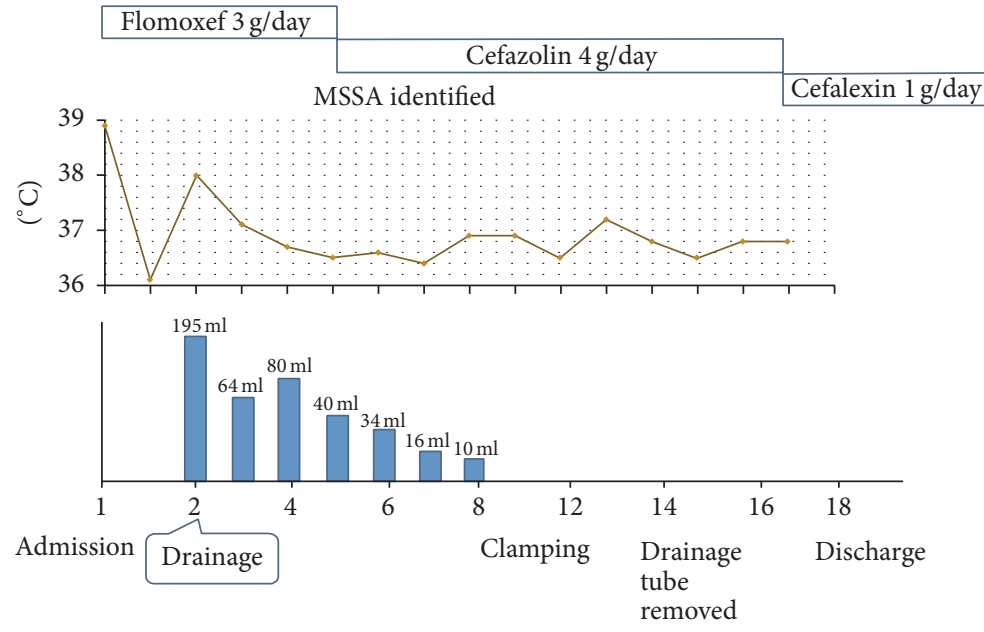

FIGURE 2: The treatment course.

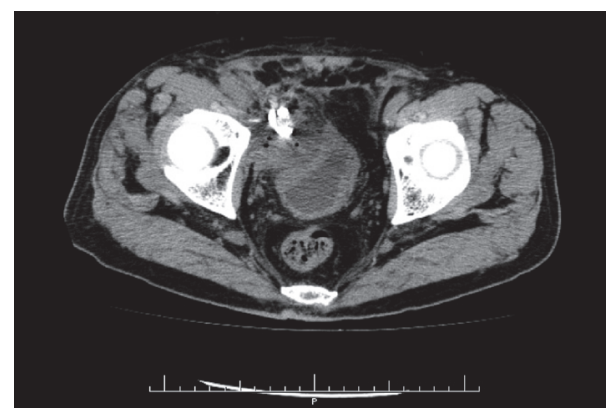

FIGURE 3: CT scan shows reduction of the lymphocele few days after clamping.

US-guided drainage of the fluid collection and aspirated purulent fluid. Gram staining of the fluid showed Grampositive cocci. Three days after drainage, we changed medication to cefazolin $4 \mathrm{~g}$ per day because methicillin-susceptible S. aureus was detected in the culture of the fluid. Seven days after drainage, his drainage gradually decreased to $10 \mathrm{ml}$, so we clamped the drainage tube. A few days after clamping, a CT scan showed reduction of the lymphocele (Figure 3), and then we removed the drainage tube. He was discharged from our hospital with treatment of cephalexin $1 \mathrm{~g}$ per day until the 15th day after drainage. A CT scan taken 2 months after discharge showed that the lymphocele had resolved. He has experienced no recurrence since then.

\section{Discussion}

Naselli et al. showed a 30\% incidence of asymptomatic lymphocele after prostatectomy regardless of surgical procedures, open surgery, or laparoscopic surgery [3]. Some study also reported that symptomatic lymphoceles developed in $2-5 \%$ of cases undergoing either open surgery or RARP [1].

The present case did not have risk factors already known for lymphocele, such as diabetes, number of lymph nodes removed, extraperitoneal approach, and the use of low molecular weight heparin, which were previously reported as factors predictive of lymphocele formation by Raheem et al. [4]. In addition, our patient did not have particular characteristics similar to those of the other cases of prostatectomy.

Keskin et al. [5] reported a quite high incidence of lymphoceles within 1 month after RARP when performing US follow-up after RARP; most of the lymphoceles (76\%) had disappeared by 3 months. However, a significant number (64\%) of the lymphoceles that did persist over 3 months after RARP became symptomatic. Therefore, they recommended that routine US imaging be performed during the first 3 months after surgery. When a lymphocele is detected by the US examination, percutaneous drainage should be considered. 


\section{Conclusion}

As many cases of prostatectomy are performed with RARP, the number of the delayed infections of lymphoceles would be increasing. Therefore, we should be aware of such a rare complication when a patient that underwent RARP with lymph node dissection presents with fever or other nonspecific symptoms.

\section{Disclosure}

This research did not receive any specific grant from funding agencies in the public, commercial, or not-for-profit sectors.

\section{Conflicts of Interest}

The authors declare that they have no conflicts of interest.

\section{References}

[1] K. C. Zorn, M. H. Katz, A. Bernstein et al., "Pelvic lymphadenectomy during robot-assisted radical prostatectomy: assessing nodal yield, perioperative outcomes, and complications," Urology, vol. 74, no. 2, pp. 296-302, 2009.

[2] M. Tremp, T. Sulser, and H.-H. Seifert, "Delayed infection of a pelvic lymphocele following robotic radical prostatectomy and pelvic lymphadenectomy: two cases," Urologia Internationalis, vol. 83, no. 4, pp. 479-481, 2009.

[3] A. Naselli, R. Andreatta, C. Introini, V. Fontana, and P. Puppo, "Predictors of symptomatic lymphocele after lymph node excision and radical prostatectomy," Urology, vol. 75, no. 3, pp. 630635, 2010.

[4] O. A. Raheem, W. M. Bazzi, J. K. Parsons, and C. J. Kane, "Management of pelvic lymphoceles following robot-assisted laparoscopic radical prostatectomy," Urology Annals, vol. 4, no. 2, pp. 111-114, 2012.

[5] M. S. Keskin, Ö. B. Argun, C. Öbek et al., "The incidence and sequela of lymphocele formation after robot-assisted extended pelvic lymph node dissection," BJU International, vol. 118, no. 1, pp. 127-131, 2016 


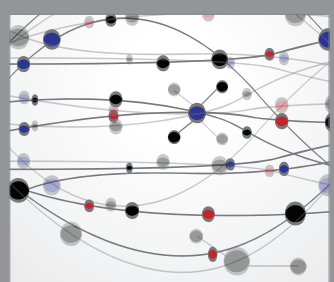

The Scientific World Journal
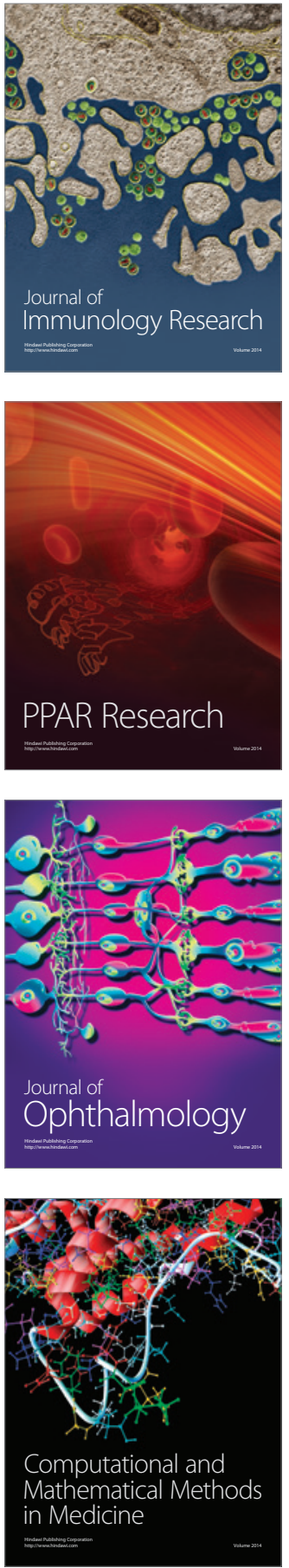

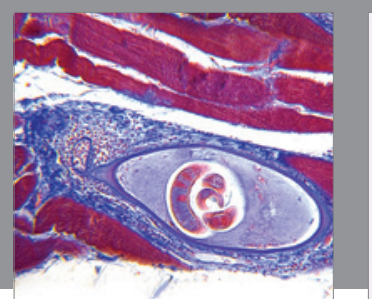

Gastroenterology Research and Practice
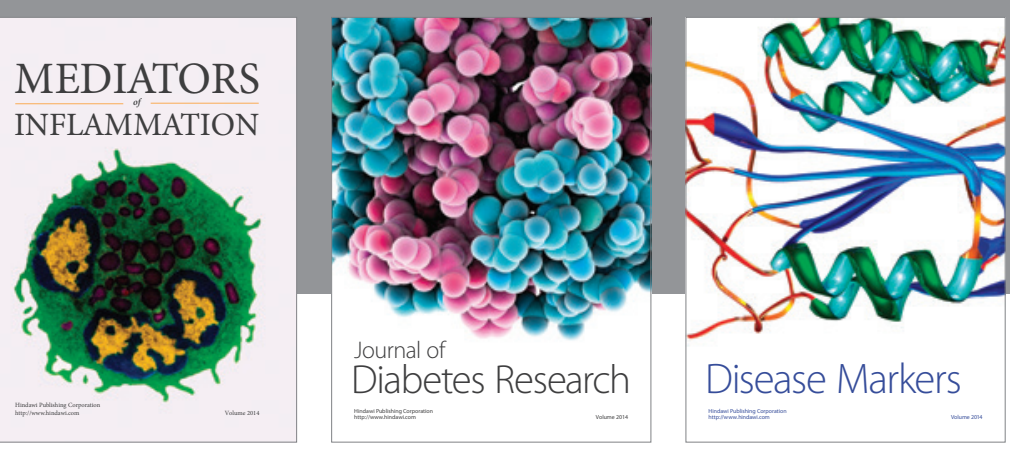

Disease Markers

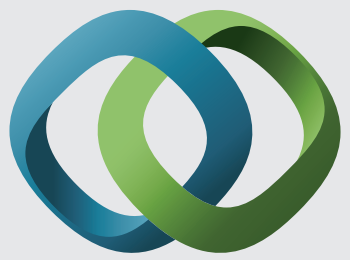

\section{Hindawi}

Submit your manuscripts at

https://www.hindawi.com
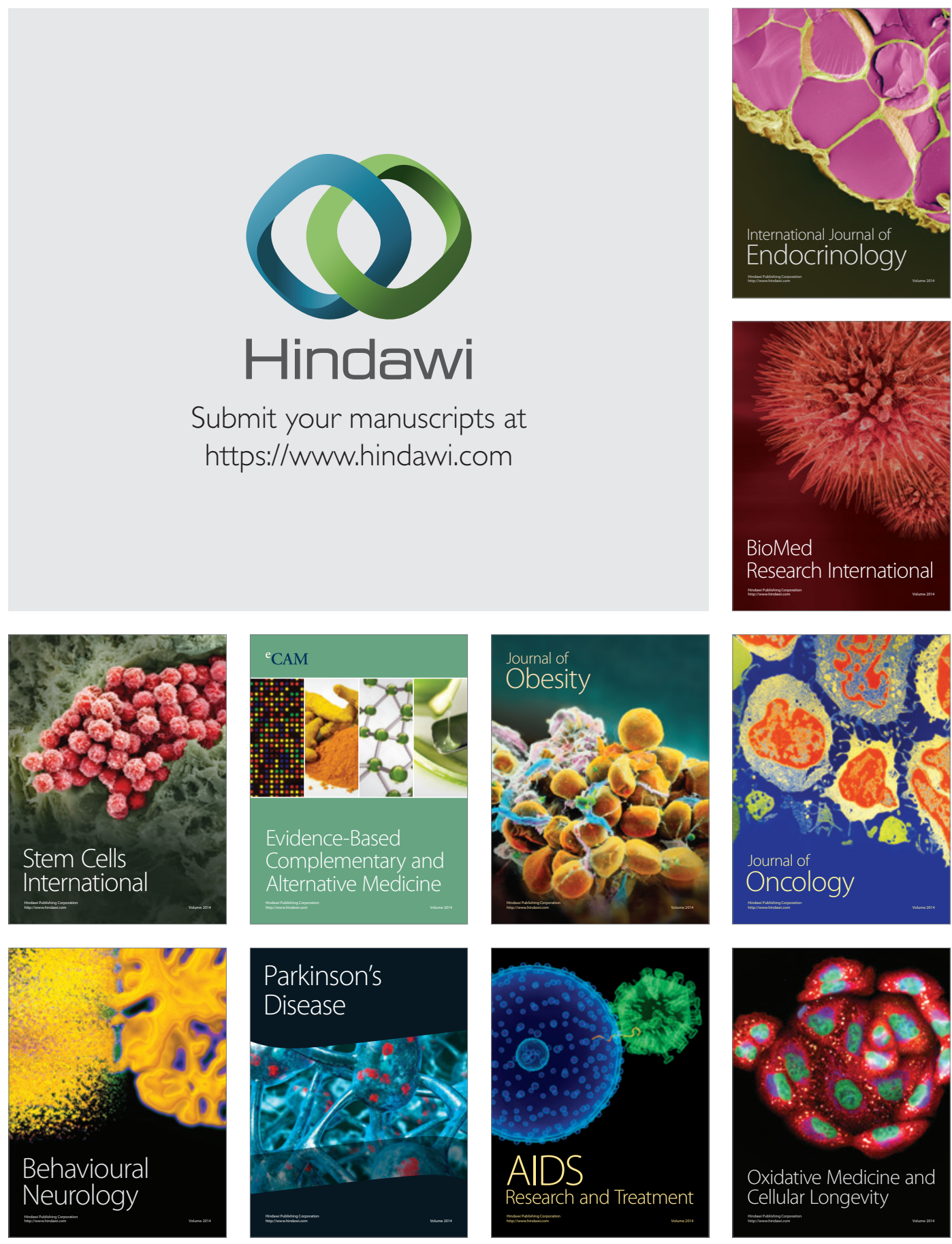